\title{
A New Constellation Configuration Scheme for Communicating Architecture in Cislunar Space
}

\author{
Ming Xu, ${ }^{1}$ Jinlong Wang, ${ }^{1}$ Shengli Liu, ${ }^{2}$ and Shijie $\mathrm{Xu}{ }^{1}$ \\ ${ }^{1}$ Department of Aerospace Engineering, School of Astronautics, Beihang University, Beijing 100191, China \\ ${ }^{2}$ R. \& D. Center, DFH Satellite Co., Ltd., Beijing 100094, China \\ Correspondence should be addressed to Ming Xu; xuming@buaa.edu.cn
}

Received 29 August 2012; Revised 2 December 2012; Accepted 10 December 2012

Academic Editor: Xing-Gang Yan

Copyright (c) 2013 Ming Xu et al. This is an open access article distributed under the Creative Commons Attribution License, which permits unrestricted use, distribution, and reproduction in any medium, provided the original work is properly cited.

\begin{abstract}
A new constellation configuration is proposed to provide near-constant communications between any two points in the cislunar space. Four satellites were allocated on some natural orbits near $L L_{2}, L L_{4}, L L_{5}$, and $L L_{3}$ points, the first three of which are expected to supply a global coverage on the Moon, and the last three afford a global coverage on the Earth. Considering the real dynamics and geometries, a full-ephemeris model is employed to refine the quasi-periodic trajectories near $L L_{2}, L L_{3}, L L_{4}$, and $L L_{5}$ points by a multiple shooting corrector numerically. The revisiting intervals between the successive revisits on specified points by the constellation are demonstrated both on the geographical maps of the Earth and the Moon, with a consideration of equatorial inclinations with respect to the Earth-Moon orbital plane. Moreover, the coverage periodicity on the Moon is induced by Metonic cycle of 19 years. Cost analysis on stationkeeping and launching is implemented for this constellation in detail. Finally, some aspects about communications, such as satellite links, delays, ground stations, and communication devices, are surveyed preliminarily.
\end{abstract}

\section{Introduction}

With the increased interests in lunar exploration and development, autonomous and manned lunar surface missions will become more and more frequent in the future decades. In particular, with the construct of lunar laboratory and lunar base, a large number of data and images need to be transmitted from Moon to Earth (or reverse). Some of the missions, for example, the manned exploration on the lunar surface, require real-time communication. The communicating architecture in cislunar space, therefore, becomes a crucial problem.

Since 1960s many scholars have contributed a lot to the design of satellite configuration architecture. An important task of the constellation design is to make satellites own some desired coverage to the surface of the expected planet. Three satellites placed on the geostationary orbits equidistantly will almost cover the surfaces of the Earth [1]. To achieve the multiple coverage, Walker [2] developed a common methodology to design the magical Walker- $\delta$ constellation, which has potential applications in the global position system (GPS). Ballard [3] designed rosette constellations with flowerlike orbital patterns to achieve invariant coverage on different deployment attitudes or orientations relative to Earth coordinates. Most of the significant works were employing Kepler orbit (or perturbed Kepler orbit) around the single planet to provide continuous communication between any points on the Earth. However, the constellation yielded in context of restricted two body problem is weak in building the link between the two separated planets, like Earth and Moon.

Because of the nature of the solutions, some useful architectures for lunar coverage may exist within the context of the restricted three-body problem (R3BP). Farquhar $[4,5]$ used a multibody model to design trajectories for communications between the far side of the Moon and the Earth. As one family of natural solution of R3BP, libration point orbits have potential uses in lunar constellation architecture to ensure adequate coverage. Chow et al. [6] considered that the most cost for the low Earth orbit constellation was the launch costs and reduced cost by using a single large launch vehicle 
to carry an entire fleet of microsatellites to the $L L_{1}$ point. Grebow [7] created architectures by quasi-periodic orbits around $L L_{1}$ and $L L_{2}$ points for continuous coverage of the south pole of the Moon with only two spacecrafts. Bhasin and Hayden [8] adopted libration point orbits in deep space communicating architecture developed for NASA's future exploration and science missions. Kulkarni et al. [9] postulated a set of satellites placed in Halo orbits at Earth-Moon, Sun-Earth, and Sun-Mars libration points and a couple of relay satellites in a heliocentric earth orbit to support data and command communication for future space missions to Moon, Mars, and beyond. Lee et al. [10, 11] proposed a conceptual constellation to substitute geostationary orbit by three satellites placed on $L L_{3}, L L_{4}$, and $L L_{5}$ points; however, they did give the feasibility and coverage analysis in detail. Grebow et al. [12] investigated some natural orbits in full ephemeris model with periods ranging from 7 to 16 days and employed two spacecrafts in combination with Earth-Moon libration point orbits to achieve constant communications for lunar south pole exploration mission.

Quite different from the researches referred above, a new constellation configuration is proposed to provide nearconstant communications between any two points in the cislunar space in this paper, with four satellites allocated on some natural orbits near $L L_{2}, L L_{4}, L L_{5}$, and $L L_{3}$ points, respectively. The first three satellites in this communicating architecture are expected to supply a global coverage on Moon, while the last three will afford a global coverage on Earth. The constellation is beneficial for the communications between different points on the grounds of Earth or Moon, even for the case with no direct links as one is located at the far side of the Moon and another is placed on the Earth.

Considering the real dynamics and geometries, a full ephemeris model is employed to refine the quasi-periodic trajectories near $L L_{2}, L L_{3}, L L_{4}$, and $L L_{5}$ points by a multiple shooting corrector numerically. The revisiting intervals between the successive revisits on specified points by the constellation are demonstrated on the geographical maps of both the Earth and the Moon, with a consideration of equatorial inclinations with respect to the Earth-Moon orbital plane. Moreover, the coverage periodicity on the Moon is induced by Metonic cycle of 19 years.

Furthermore, cost analysis on stationkeeping and launching is implemented in detail in full ephemeris model. According to the stationkeeping and inserting strategies proposed in this paper, the total inserting cost of this constellation is $14.4731 \mathrm{~km} / \mathrm{s}$, and the total stationkeeping cost of this constellation is $261.8 \mathrm{~m} / \mathrm{s} /$ year.

Compared with two geostationary orbit constellations that require six spacecrafts to cover both the Earth and Moon, this constellation architecture saves two spacecrafts to reduce the cost of the system a lot, which is considered as an alternative of limited geostationary orbit resources.

\section{Design of Constellation Architecture}

2.1. Constellation Configuration Scheme. To describe the global coverage constellation in cislunar space, four different coordinates are referred to in this paper, as the syzygy frame in Earth-Moon system, the Earth-centered fixed frame, (i.e., WGS-84 frame), the Moon-centered fixed frame, and the Earth-centered inertial frame (i.e., J2000 frame), where the syzygy frame has the following features: the origin $O$ is fixed at the barycenter of the Earth-Moon system; the $z$-axis is perpendicular to the lunar orbital plane; the $x$-axis points from Earth to Moon; and the $y$-axis is determined by the right-hand side rule.

Ignored other celestial bodies' gravitation and eccentricity of lunar orbit, the dynamics of system consisted of spacecraft, Earth, and Moon can be regarded as the circular restricted three-body problem (CR3BP). There are five libration points fixed in the syzygy frame, labeled as $L L_{i}, i=$ $1, \ldots, 5$, where $L L_{2}$ and $L L_{3}$ locate at the far side of the Moon and Earth, respectively, and $L L_{4}$ and $L L_{5}$ form an equilateral triangle with Earth and Moon.

The combination of $L L_{2}, L L_{4}, L L_{5}$, and $L L_{3}$ shapes a double-triangle configuration, where the first three points product one triangle focusing at the Moon, while the last three form the other triangle centering the Earth. By the inspiration of the global coverage constellation created by three satellites equispaced on the geostationary orbit, a new communicating architecture is proposed to provide some links (with time delay of 2-3 sec) between any two points in the cislunar space by $L L_{2}, L L_{4}, L L_{5}$, and $L L_{3}$ points, shown in Figure 1. With the help of this constellation system, a direct or indirect link exists for any two points in cislunar space, even for the two points $P_{M}$ and $P_{E}$ located on the far side of Earth and Moon respectively (one of the feasible indirect links is as follows: $P_{M} \rightarrow L L_{2} \rightarrow L L_{4} \rightarrow L L_{3} \rightarrow P_{E}$ ). Moreover, the quasi-halo orbit near $L L_{2}$ inheriting from halo family is employed to avoid some links from $L L_{2}$ point to Earth blocked by the Moon.

\subsection{Numerical Refinements by Multiple Shooting Corrector.} The eccentricity of the Moon's orbit and gravitational forces from other celestial bodies (like the Sun and the Jupiter) will make CR3BP different from the real dynamics, even the libration point cannot keep stationary due to this perturbations.

Therefore, a multiple shooting corrector is employed to create some natural orbits near the libration points in the full ephemeris model, which is useful for the computation of highly unstable periodic orbits with very long periods, as $J_{2}$ invariant relative orbits $[13,14]$ and solar sail's displaced orbits [15].

The general idea is to use a multiple shooting method similar to the one used for the numerical solution of boundary value problems. As in the iteration procedure, the total time span $\left[t_{0}, t_{f}\right]$ is split into a number of shorter subintervals by $N$ equispaced points $t_{0}, t_{1}, t_{2}, \ldots, t_{N-1}, t_{f}$. Denote the subinterval with equal length as $\Delta t=t_{i+1}-t_{i}(i=0,1,2, \ldots, N-$ 1 ), and denote the point on the quasi-periodic trajectory as $\mathbf{Q}_{i}=\left[t_{i}, x_{i}, y_{i}, z_{i}, \dot{x}_{i}, \dot{y}_{i}, \dot{z}_{i}\right]^{T}(i=0,1,2, \ldots, N-1)$. Let $\phi\left(\mathbf{Q}_{i}\right)$ be the image of the point $\mathbf{Q}_{i}$ under the flow associated to the full ephemeris dynamics after an amount of time $\Delta t$. To keep all the points $\mathbf{Q}_{i}$ staying on the same trajectory solved from 


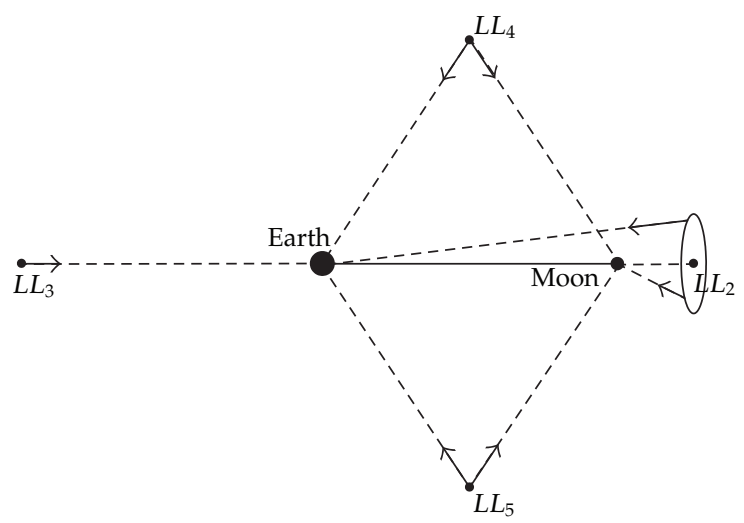

FIGURE 1: The communicating constellation architecture in cislunar space. $L L_{2}, L L_{4}$, and $L L_{5}$ form one triangle to supply a global coverage on Moon; $L L_{3}, L L_{4}$, and $L L_{5}$ form the other triangle points to supply a global coverage on Earth.

the dynamics, some corrections to $\mathbf{Q}_{i}$ are required to fulfill the matching conditions, as

$$
\mathbf{F}\left(\begin{array}{c}
\mathbf{Q}_{1} \\
\mathbf{Q}_{2} \\
\vdots \\
\mathbf{Q}_{N}
\end{array}\right)=\left(\begin{array}{c}
\phi\left(\mathbf{Q}_{1}\right) \\
\phi\left(\mathbf{Q}_{2}\right) \\
\vdots \\
\phi\left(\mathbf{Q}_{N-1}\right)
\end{array}\right)-\left(\begin{array}{c}
\mathbf{Q}_{2} \\
\mathbf{Q}_{3} \\
\vdots \\
\mathbf{Q}_{N}
\end{array}\right)=0
$$

In the $j$ th iteration of this procedure, the matching equation can be written as

$$
\left.\frac{\partial \mathbf{F}}{\partial \mathbf{Q}}\right|_{\mathbf{Q}^{(j)}} \cdot\left[\mathbf{Q}^{(j+1)}-\mathbf{Q}^{(j)}\right]=-\left.\mathbf{F}\right|_{\mathbf{Q}^{(j)}}
$$

where $\mathbf{Q}^{(j)}=\left[\mathbf{Q}_{1}^{(j)} \mathbf{Q}_{2}^{(j)} \ldots{ }_{N}^{(j)}\right]^{T}$. The differential function of F has the following expression:

$$
\frac{\partial \mathbf{F}}{\partial \mathbf{Q}}=\left(\begin{array}{ccccc}
\left.\frac{\partial \varphi}{\partial \mathbf{Q}}\right|_{\mathbf{Q}_{1}^{(j)}} & -\mathbf{I} & & & \\
& \left.\frac{\partial \varphi}{\partial \mathbf{Q}}\right|_{\mathbf{Q}_{2}^{(j)}} & -\mathbf{I} & & \\
& & \ddots & \ddots & \\
& & & \left.\frac{\partial \varphi}{\partial \mathbf{Q}}\right|_{\mathbf{Q}_{N-1}^{(j)}} & -\mathbf{I}
\end{array}\right) .
$$

The iterations have been included in the software package OrbitalPredictor developed by Beihang University [16], which adapts JPL ephemeris model to generate some natural orbits near the libration points.

To accelerate the convergence of the algorithm, an initial iteration to the natural solution of the dynamics is required. For $L L_{3}, L L_{4}$, and $L L_{5}$, the libration points themselves can work as the initial iterations to create bounded trajectories near these points; however, for a quasi-halo orbit near $L L_{2}$ point, an effective initial iteration can employ the following Richardson expansion developed from the LindstedtPoincaré method [17, 18]:

$$
\begin{aligned}
x= & a_{21} A_{x}^{2}+a_{22} A_{z}^{2}-A_{x} \cos \omega t+\left(a_{23} A_{x}^{2}-a_{24} A_{z}^{2}\right) \cos 2 \omega t \\
& +\left(a_{31} A_{z}^{3}-a_{32} A_{x} A_{z}^{2}\right) \cos 3 \omega t, \\
y= & \kappa A_{x} \sin \omega t+\left(b_{21} A_{x}^{2}-b_{22} A_{z}^{2}\right) \sin 2 \omega t \\
& +\left(b_{31} A_{x}^{3}-b_{32} A_{x} A_{z}^{2}\right) \sin 3 \omega t, \\
x= & \pm A_{z} \cos \omega t \pm d_{21} A_{z}[\cos 2 \omega t-3] \\
& \pm\left(d_{32} A_{z} A_{x}^{2}-d_{31} A_{z}^{3}\right) \cos 3 \omega t,
\end{aligned}
$$

where the detailed discussion on the coefficients $a, b, c, \omega$, and $\kappa$ can be found in [17]. To keep the frequencies in $z$-axis equal to $x$ - and $y$-axes, the amplitudes $A_{x}$ and $A_{z}$ are dependent on each other and also need to be no less than some critical values. Therefore, the amplitude $A_{x}$ can be selected as the only parameter to identify different halo orbits, and then a halo orbit with the minimal amplitude $A_{x}=11500 \mathrm{~km}$ is created by the iteration algorithm in this paper.

Following the initial iterations proposed for $L L_{2}, L L_{3}$, $L L_{4}$, and $L L_{5}$ referred to above, some bounded trajectories can be refined by the OrbitalPredictor full-ephemeris model shown in Figure 2 and then are targeted in Satellite Tool Kit (STK) using Astrogator tool $[12,19]$, which are shown in Figure 3 by STK.

\section{Coverage Analysis of Constellation}

The revisiting time is employed to parameterize the coverage of this constellation architecture, which is defined as the time intervals between the successive revisits on the specified ground point on the Earth or Moon. Therefore, this concept is measuring the interruption durations when communicating with any point. 

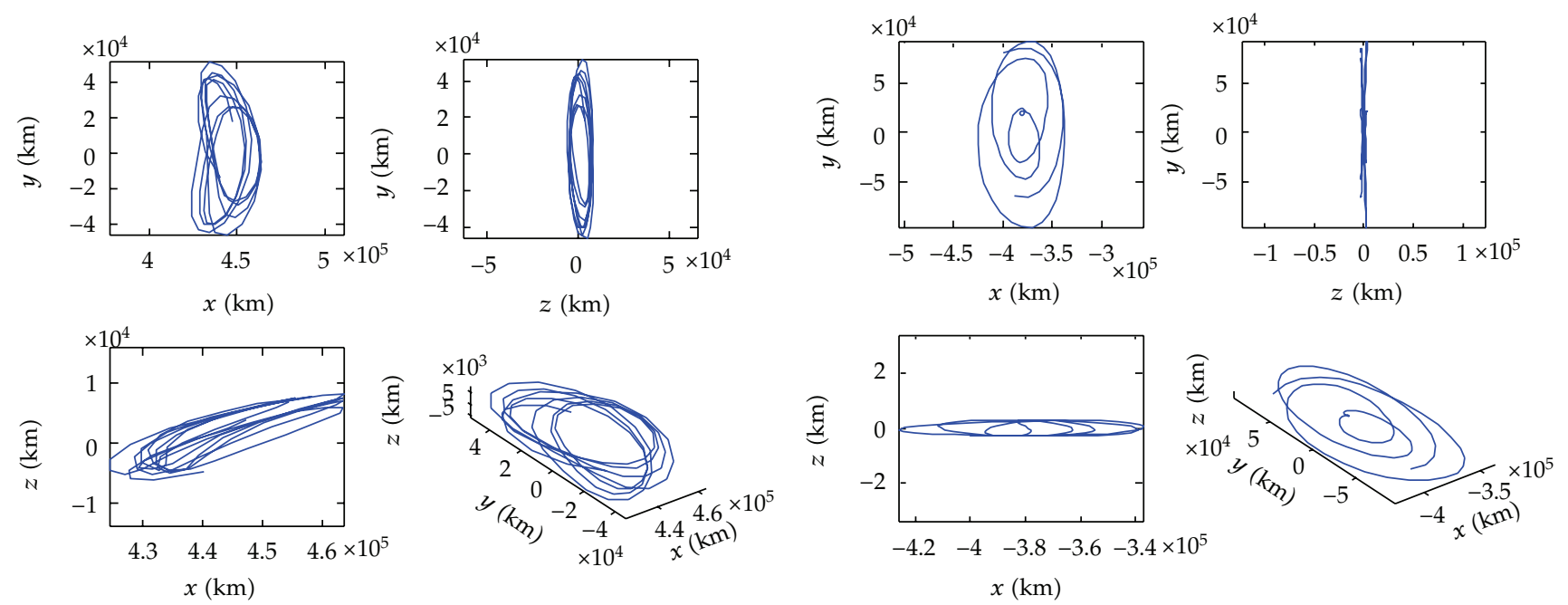

(a)

(b)
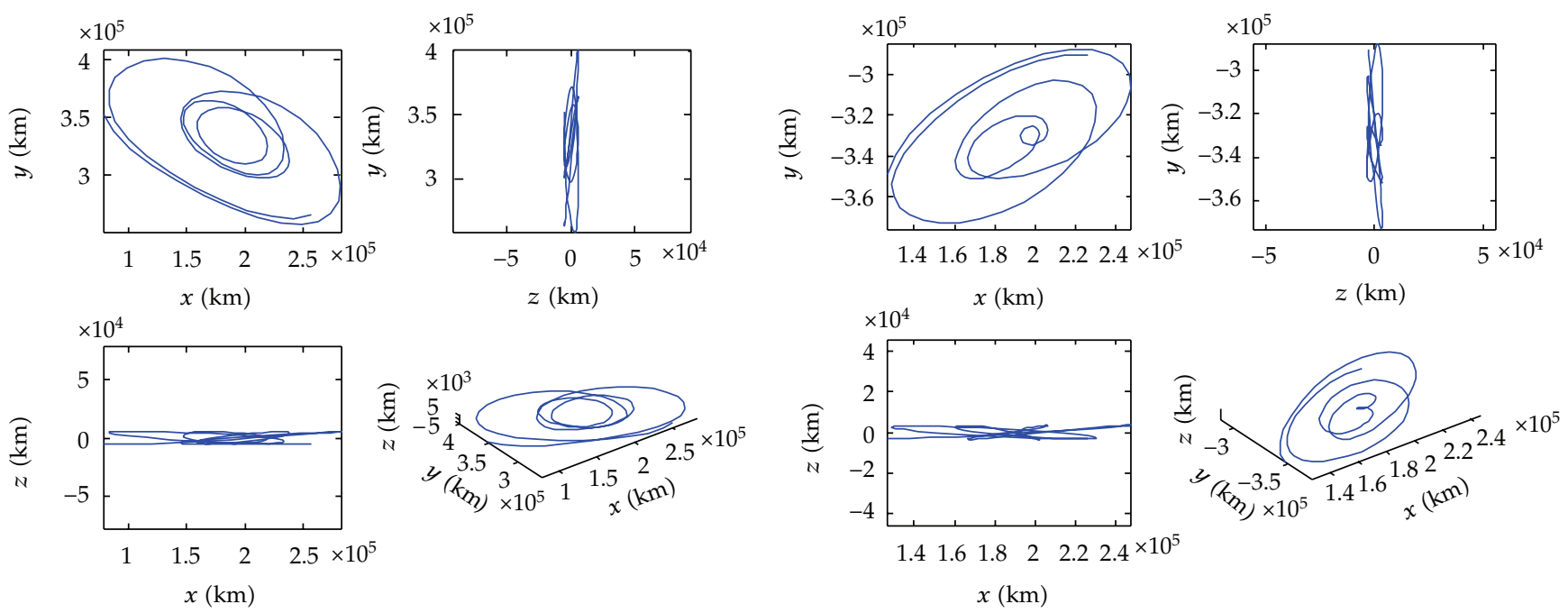

(c)

(d)

FIgURE 2: The bounded trajectories near $L L_{2}, L L_{3}, L L_{4}$, and $L L_{5}$ created by OrbitalPredictor with the epoch time of 12:00, July 1, 2015. The quasi-periodic trajectories near (a) $L L_{2}$ point, (b) $L L_{3}$ point, (c) $L L_{4}$ point, and (d) $L L_{5}$ point.

3.1. Coverage to Moon by the Constellation. As the preliminary investigation on the coverage to Moon, some hypotheses can be adopted to plot the covering region, including the following: the inclination of the equator plane with respect to the lunar orbital plane is neglected as $0^{\circ}$, instead of its true value of $6^{\circ} 41^{\prime}$; the eccentricity of lunar orbit and the gravitation from the Sun are ignored to place the spacecrafts on $L L_{3}, L L_{4}$, and $L L_{5}$ points and the halo orbit near $L L_{2}$ without any stationkeeping maneuver; that is, the libration point orbits are yielded from CR3BP. Due to the osculating position on halo orbit, the maximal and common regions are achieved in Figure 4, where the former is defined as the region covered by the constellation at any time, while the latter is defined as the region covered all the time. Therefore, the constellation can cover the lunar surface by $99.45 \%$ (maximal region) and $98.95 \%$ (common region) except the south and north poles.
In a full investigation on the coverage to Moon, no hypothesis is employed, including the real geometries and the full dynamics. All the bounded trajectories in this constellation are refined by the OrbitalPredictor full-ephemeris model, and the revisiting intervals are counted statistically in real geometrical scenario by STK.

The Moon is in synchronous rotation: it rotates about its axis in about the same time it takes to orbit the Earth; however, due to the inclination between lunar orbital and equator planes and some others, there are several librations to change the angle from which the Moon is seen, allowing about $59 \%$ of its surface to be seen from the Earth.

Moreover, the 19-year Metonic cycle of the equatorial inclination, with respect to lunar orbital plane, makes the Moon go overhead at latitudes over the equator from $18.5^{\circ}$ to $28.5^{\circ}$, so it is necessary to give results in term of time in a whole cycle. Figure 5 depicts the revisiting times on the lunar 


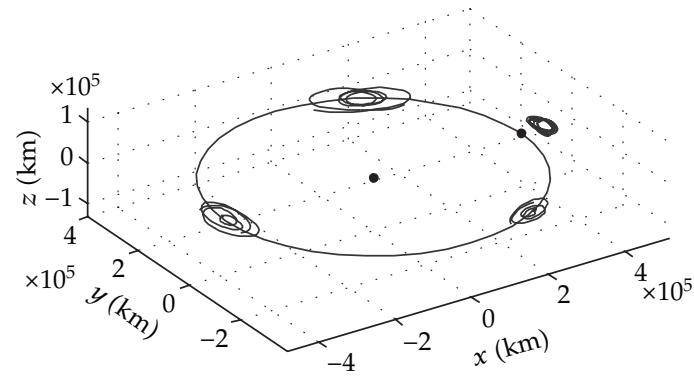

(a)

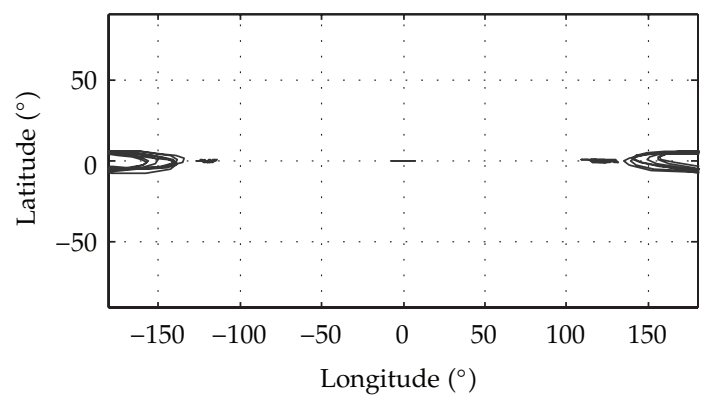

(c)

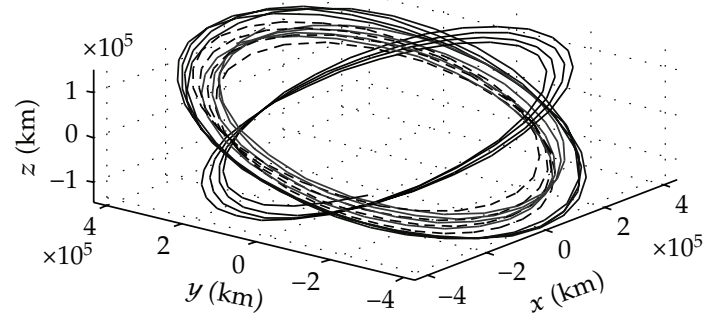

(b)

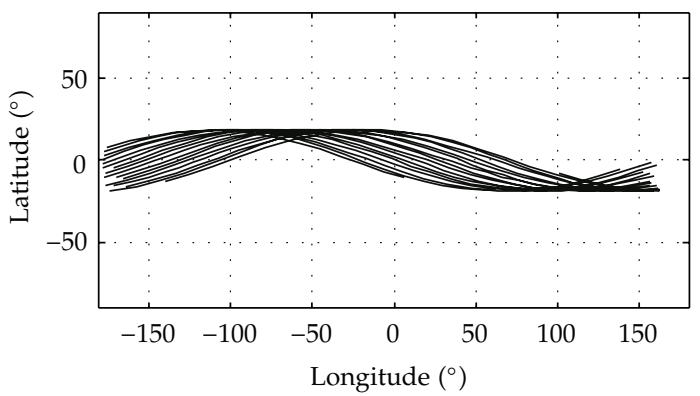

(d)

FIGURE 3: The quasi-periodic trajectories near $L L_{2}, L L_{3}, L L_{4}$, and $L L_{5}$ created by STK with the epoch time of 12:00, July 1, 2015. The bounded trajectories are shown in (a) the syzygy frame in Earth-Moon system and (b) the Earth-centered inertial frame. The ground tracks of the trajectories are shown in (c) the Earth-centered fixed frames and (d) the Moon-centered fixed frames.

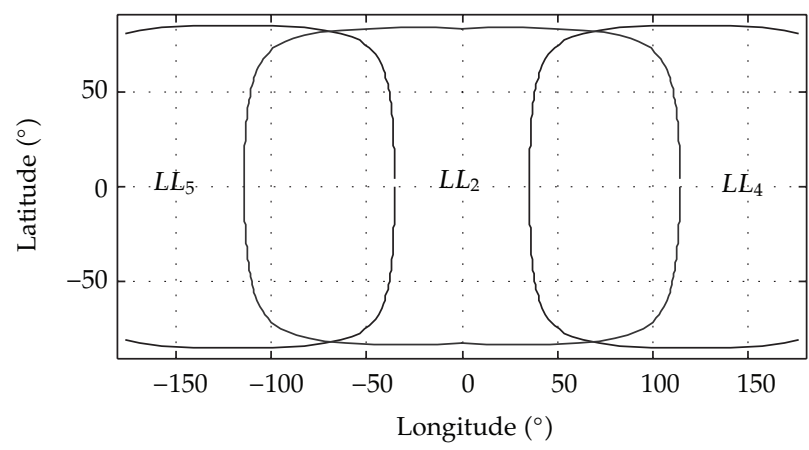

(a)

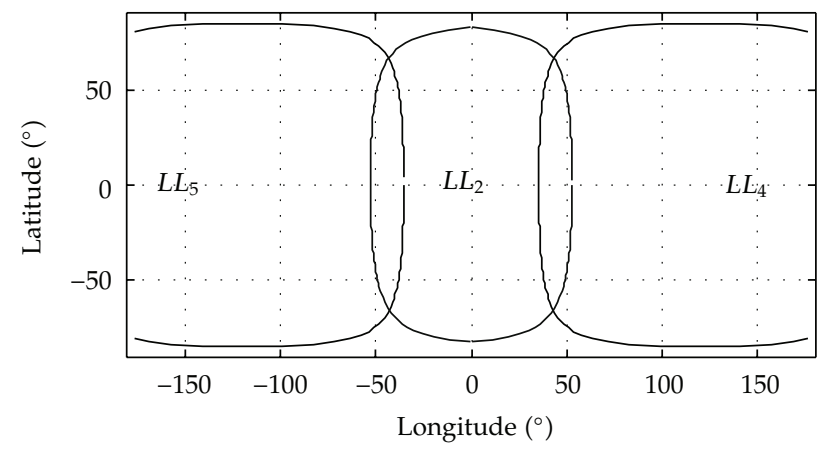

(b)

FIGURE 4: The preliminary analysis on covering region by the constellation on the lunar surface. (a) The maximal region covered by the constellation at any time; (b) the common region covered by the constellation all the time.

surface by the constellations created from different epoch times in a Metonic cycle. The maximal coverage for full geometries is investigated by the maximal revisiting times during this cycle on the specified point; however, the common coverage is yielded by the minimal revisiting times during this cycle, and then both the maximal and minimal revisiting times are revealed in Figure 6 on the global surface.

Different from the preliminary results, some conclusions can be induced from Figures 5 and 6 . Firstly, all the points on the lunar surface can be covered by this constellation, where most of them are accessed without any time interval (i.e., the blank regions in the top of Figure 6), and few of them are revisited after some intervals less than 5 days (i.e., the colored regions in the top of Figure 6).

Secondly, due to the lunar synchronous rotation, this constellation is weak in covering some longitude lines located near $150^{\circ} \mathrm{W}$ and $150^{\circ} \mathrm{E}$ shown in Figure 5. The discontinuous communications are interrupted by some intervals less than 2 hours.

Thirdly, only some sites keep discontinuous communications during a whole Metonic cycle, which are marked in the bottom of Figure 6 from $20^{\circ} \mathrm{N} 140^{\circ} \mathrm{W}$ to $70^{\circ} \mathrm{N} 140^{\circ} \mathrm{W}$, from $30^{\circ} \mathrm{S} 150^{\circ} \mathrm{W}$ to $2^{\circ} \mathrm{N} 150^{\circ} \mathrm{W}$, from $85^{\circ} \mathrm{S} 55^{\circ} \mathrm{E}$ to $85^{\circ} \mathrm{S} 100^{\circ} \mathrm{E}$, and from $80^{\circ} \mathrm{S} 170^{\circ} \mathrm{E}$ to $80^{\circ} \mathrm{S} 130^{\circ} \mathrm{W}$. 


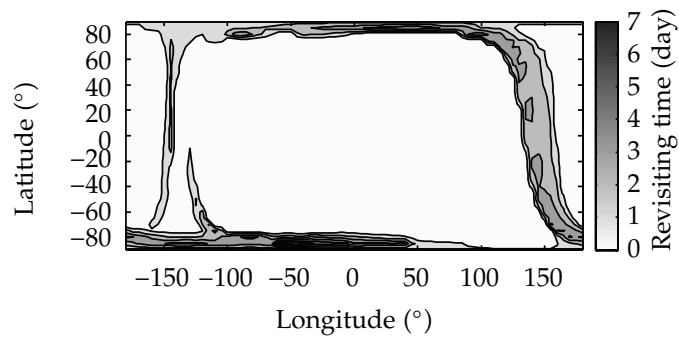

(a) Epoch time: 12:00, July 1, 2015

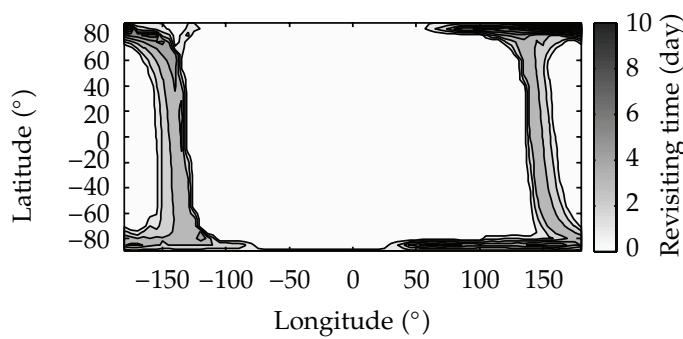

(c) Epoch time: 12:00, July 1, 2021

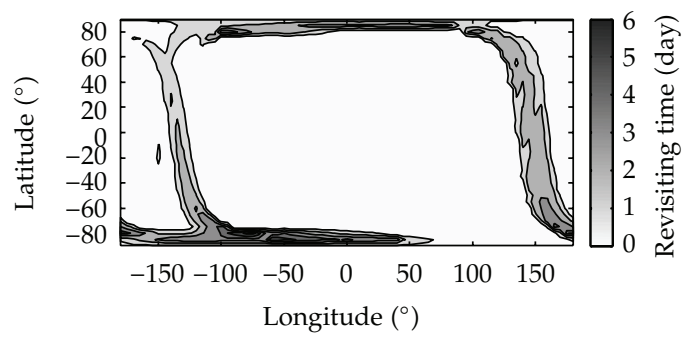

(e) Epoch time: 12:00, July 1, 2027

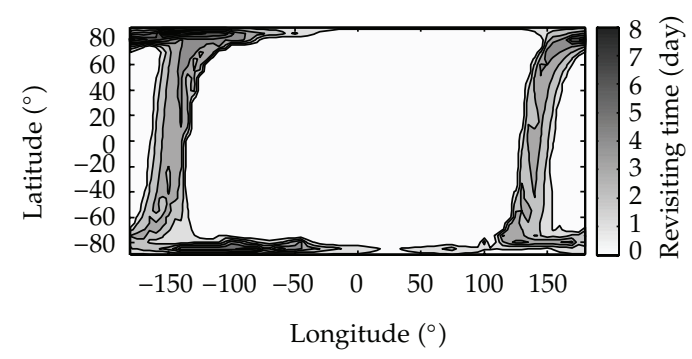

(b) Epoch time: 12:00, July 1, 2018

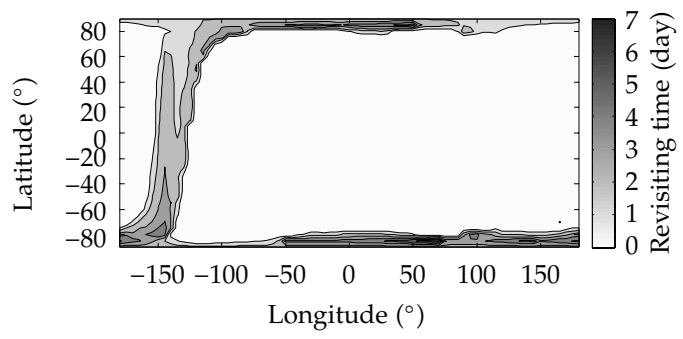

(d) Epoch time: 12:00, July 1, 2024

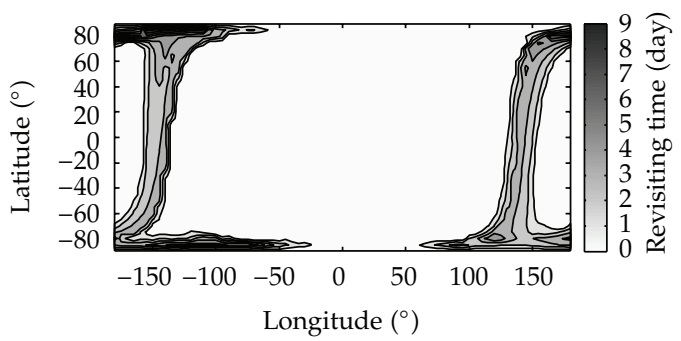

(f) Epoch time: 12:00, July 1, 2030

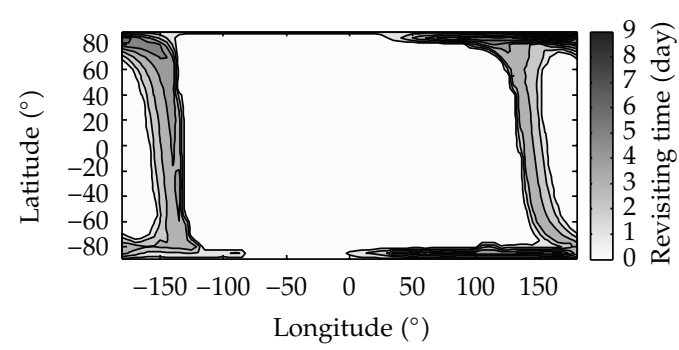

(g) Epoch time: 12:00, July 1, 2033

Figure 5: The full analysis on revisiting time on the lunar surface during a whole Metonic cycle from 2015 to 2034 . The coverage analyses are implemented by the epoch times of (a) 12:00, July 1, 2015, (b) 12:00, July 1, 2018, (c) 12:00, July 1, 2021, (d) 12:00, July 1, 2024, (e) 12:00, July 1, 2027, (f) 12:00, July 1, 2030, and (g) 12:00, July 1, 2033.

Fourthly, the worst intervals occur at the high-latitude areas instead of the poles because the maximal revisiting time is 0.96 day at the north pole while 1.21 days at the south pole. Thus this constellation has a good coverage performance at the poles.

3.2. Coverage to Earth by the Constellation. As the preliminary investigation on the coverage, the irrotational Earth is assumed in combination with no consideration of the eccentricity of lunar orbit and the gravitation from the Sun. Therefore, the constellation has the fixed configuration in the syzygy frame, which results in the maximal coverage region equal to the common coverage region shown in Figure 7. From the hypothesis referred above, the constellation can cover the Earth's surface by $99.1 \%$ except the south and north poles.

In a full investigation on the coverage to Earth, the hypothesis is unloaded with some considerations of the real geometries and full dynamics. All the quasi-periodic trajectories near $L L_{3}, L L_{4}$, and $L L_{5}$ are refined by the OrbitalPredictor full-ephemeris model, and the revisiting intervals are counted statistically in real geometrical scenario by STK.

The 19-year Metonic cycle of the equatorial inclination, with respect to lunar orbital plane, has also an influence 


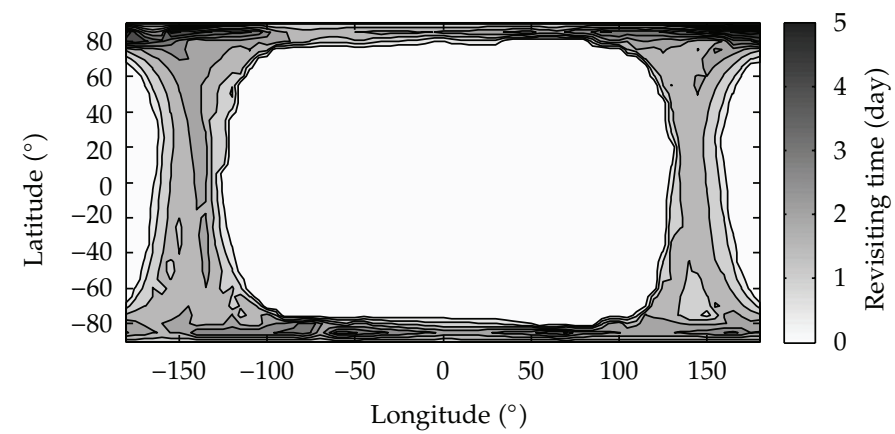

(a)

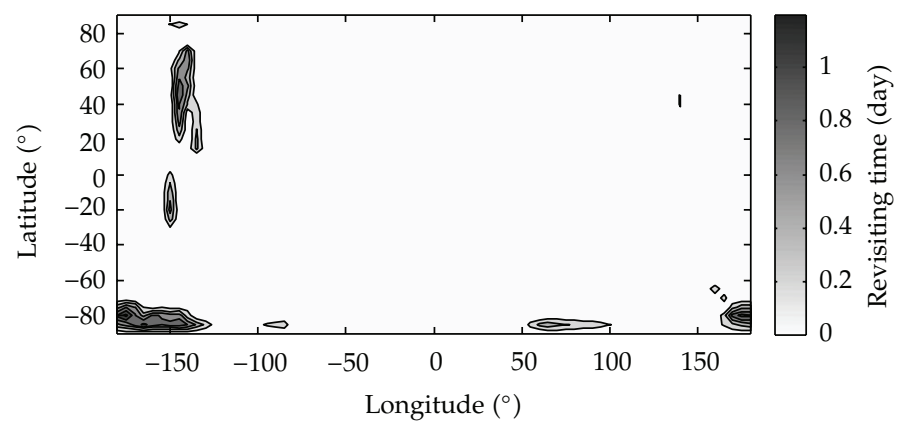

(b)

FIgURE 6: The full analysis on the maximal and common coverages during a whole Metonic cycle from 2015 to 2034 . The maximal revisiting times on the global surface during a whole Metonic cycle are implemented in (a), and the minimal revisiting times in (b).

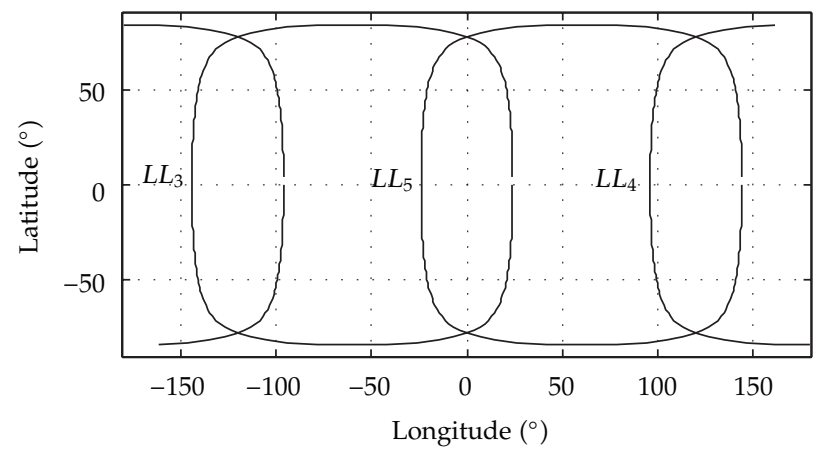

FIGURE 7: The preliminary analysis on covering region by the constellation on the Earth's surface.

on the coverage by the constellation. However, because of rotating more quickly around its own axis, the Earth owns the constant revisiting time on the same latitude lines located near $70^{\circ} \mathrm{S}$ and $70^{\circ} \mathrm{N}$ shown in Figure 8, where the discontinuous communications are interrupted by some intervals less than 2.5 hours. It is quite different from the Moon where the discontinuous communications are just locating on the longitude lines near $150^{\circ} \mathrm{W}$ and $150^{\circ} \mathrm{E}$. Moreover, the regions on other latitudes are accessed without any time interval (i.e., the blank regions in the top of Figure 8).

In addition, the conclusion that the revisiting time keeps invariant on the same latitude line will be true for a whole Metonic cycle. Therefore, the relationship between revisiting time and the latitudes will be investigated in the following section just on the longitude line of $0^{\circ} \mathrm{E}$, shown in Figures 9 and 10 .
Different from the preliminary results, some conclusions can be induced from Figures 9 and 10. Firstly, all the points on the Earth's surface can be covered by this constellation, and the discontinuous communications keep the invariant revisiting time on the same latitudes during a whole Metonic cycle.

Secondly, due to the rotation of the Earth, few of the regions located at the latitudes from $80^{\circ} \mathrm{S}$ to $52^{\circ} \mathrm{S}$ and from $55^{\circ} \mathrm{N}$ to $79^{\circ} \mathrm{N}$ are revisited after some intervals less than 2.5 hours, while the other regions are accessed without any time interval.

Thirdly, the worst intervals occur at the regions located from $71^{\circ} \mathrm{S}$ to $65^{\circ} \mathrm{S}$ and from $62^{\circ} \mathrm{N}$ to $70^{\circ} \mathrm{N}$, which keep discontinuous communications during a whole Metonic cycle. Moreover, this constellation has a good coverage performance at the poles with no time interval. 
TABLE 1: Stationkeeping cost of the constellation from 12:00, July 1, 2015, to 12:00, July 1, 2016.

\begin{tabular}{lcccc}
\hline Libration point & Number of maneuvers & Position error $(\mathrm{km})$ & Average $\Delta V(\mathrm{~m} / \mathrm{s})$ & Total $\Delta V(\mathrm{~m} / \mathrm{s})$ \\
\hline$L L_{2}$ & 52 & 954.2 & 1.96 & 1.7 \\
$L L_{3}$ & 52 & 1102.4 & 0.6 & 88.5 \\
$L L_{4}$ & 52 & 1464.6 & 0.77 & 31.2 \\
$L L_{5}$ & 52 & 1398.7 & 39.8 \\
\hline
\end{tabular}

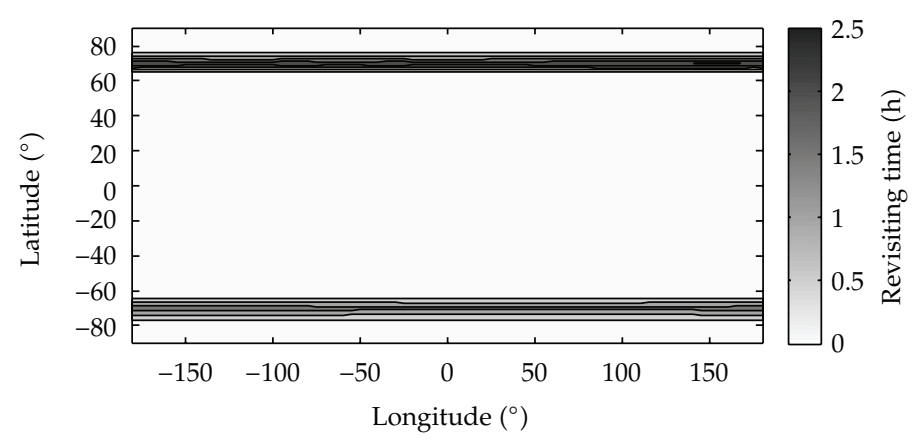

FIGURE 8: The full analysis on revisiting time on the Earth's surface with the epoch time of 12:00, July 1, 2015. The conclusion that the revisiting time keeps invariant on the same latitude line will be true for a whole Metonic cycle.

3.3. Multiple Links to Earth and Moon by $L L_{4}$ and $L L_{5}$. In this constellation, the $L L_{4}$ point takes simultaneously charge in the communications with Earth and Moon, as well as $L L_{5}$. Therefore, two communication devices need to be equipped on the spacecrafts, with their antennas pointing to the Earth and Moon, respectively. However, due to the Metonic cycle of the equatorial inclination with respect to lunar orbital plane, the boundaries of the angle between the two antennas are illustrated points in Figure 11, where the angle between the two antennas near $L L_{4}$ point varies from $47^{\circ}$ to $81^{\circ}$, and from $34^{\circ}$ to $85^{\circ}$ near $L L_{5}$ point.

\section{Cost Analysis of Constellation}

4.1. Stationkeeping Strategies and Costs. Stationkeeping correction is necessary because the true trajectory will diverge from the nominal one under the effects of the navigation error $E_{1}$, the initial error from the launch rocket $E_{2}$, and the error from inserting the nominal trajectory on velocity $E_{3}[20,21]$. However, the presence of the trajectory correction will cause velocity errors $E_{4}$. These error resources are assumed to be Gauss distributed and their average values are zero. $E_{1}$ and $E_{2}$ have the same position error variances of $10 \mathrm{~km}$ and velocity error variances of $0.01 \mathrm{~m} / \mathrm{s}(1 \sigma) ; E_{3}$ and $E_{4}$ have the velocity error variances of $3 \%$ and their velocity errors in the direction of radius are $2.06^{\circ}(3 \sigma)[22,23]$. The velocity error in the normal direction is uniformly distributed.

Assume that there are $k$ corrections during the flight on nominal trajectory, which is divided into $k+1$ sections according to the amount of corrections, as it is shown in Figure 12 .

The state errors between the actual and nominal trajectories at time $\bar{t}_{m}$ are $\delta \mathbf{R}_{m}$ and $\delta \mathbf{V}_{m}$ from the errors $E_{1}, E_{2}, E_{3}$, and $E_{4}$, respectively, and the Taylor expansion of the position vector at time $\bar{t}_{m}$ is obtained

$$
\begin{aligned}
\mathbf{R}\left(\bar{t}_{m+1}, \overline{\mathbf{R}}_{m}+\delta \mathbf{R}_{m}, \overline{\mathbf{V}}_{m}+\delta \mathbf{V}_{m}+\Delta \mathbf{V}_{m}, \bar{t}_{m}\right) \\
=\mathbf{R}\left(\bar{t}_{m+1}, \overline{\mathbf{R}}_{m}, \overline{\mathbf{V}}_{m}, \bar{t}_{m}\right)+\frac{\partial \overline{\mathbf{R}}_{m+1}}{\partial \overline{\mathbf{R}}_{m}} \cdot \delta \mathbf{R}_{m}+\frac{\partial \overline{\mathbf{R}}_{m+1}}{\partial \overline{\mathbf{V}}_{m}} \\
\cdot\left(\delta \mathbf{V}_{m}+\Delta \mathbf{V}_{m}\right)
\end{aligned}
$$

where the correction velocity $\Delta \mathbf{V}_{m}$ is used to correct the trajectory aiming at achieving the wanted position $\mathbf{R}\left(\bar{t}_{m+1}, \overline{\mathbf{R}}_{m}, \overline{\mathbf{V}}_{m}, \bar{t}_{m}\right)$ at time $\bar{t}_{m}$. According to (5), $\Delta \mathbf{V}_{m}$ can be obtained

$$
\Delta \mathbf{V}_{m}=-\left(\frac{\partial \overline{\mathbf{R}}_{m+1}}{\partial \overline{\mathbf{V}}_{m}}\right)^{-1} \cdot \frac{\partial \overline{\mathbf{R}}_{m+1}}{\partial \overline{\mathbf{R}}_{m}} \cdot \delta \mathbf{R}_{m}-\delta \mathbf{V}_{m}
$$

The correction velocity $\Delta \mathbf{V}_{m}$ is a linear comparison of $\delta \mathbf{R}_{m}$ and $\delta \mathbf{V}_{m}$, and this characteristic is definitely suitable for trajectory correction during the flight.

A correction schedule with an equi-interval correction time of 7 days is adopted in this paper to investigate the stationkeeping costs for this constellation in the full ephemeris model. The maneuver results are obtained in Table 1 for one complete year. Therefore, the total stationkeeping cost of this constellation is $261.8 \mathrm{~m} / \mathrm{s} /$ year, which is accepted for the aerospace engineering.

Furthermore, some optimization technique to decrease the stationkeeping cost can be implemented in the future, including some selections for the correction schedule, and the application of the stochastic optimal control into the correction strategy. 


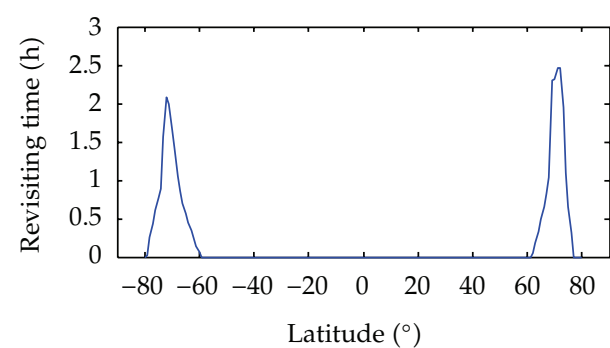

(a) Epoch time: 12:00, July 1, 2015

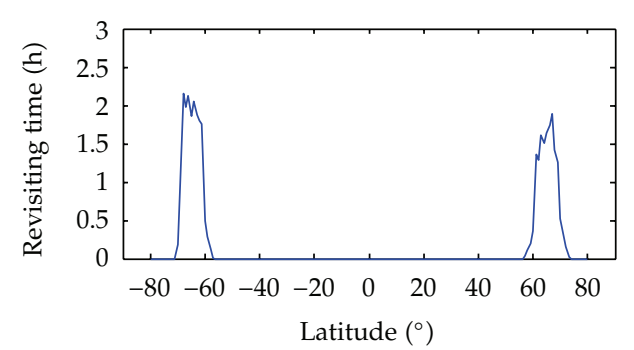

(c) Epoch time: 12:00, July 1, 2021

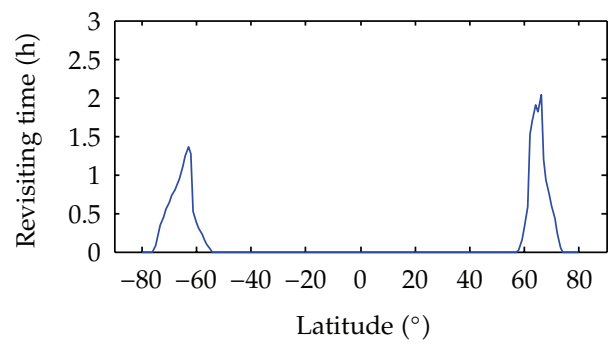

(e) Epoch time: 12:00, July 1, 2027

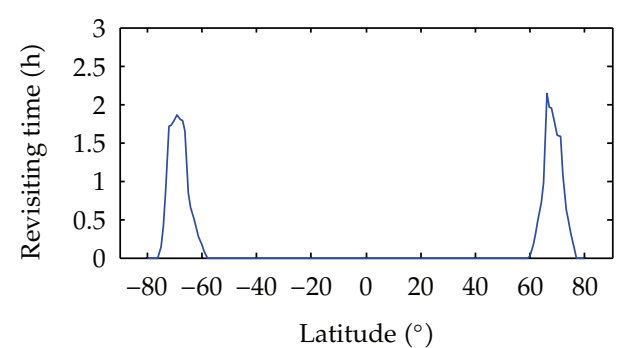

(b) Epoch time: 12:00, July 1, 2018

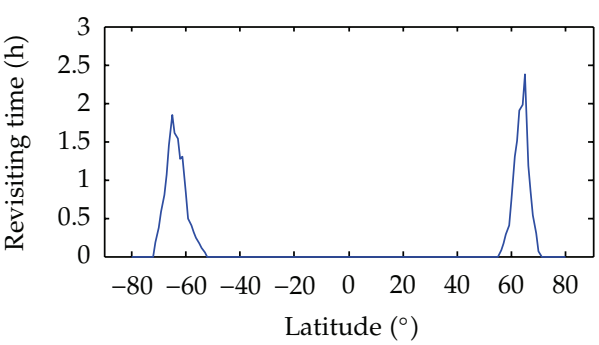

(d) Epoch time: 12:00, July 1, 2024

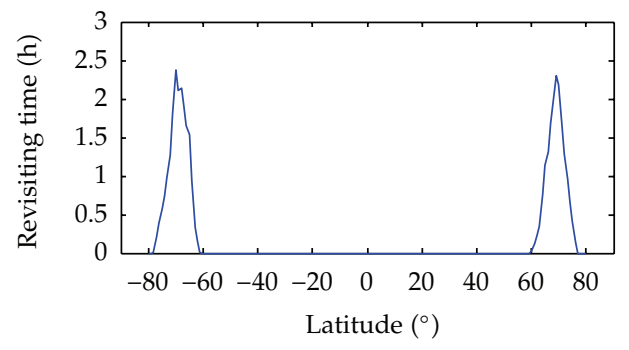

(f) Epoch time: 12:00, July 1, 2030

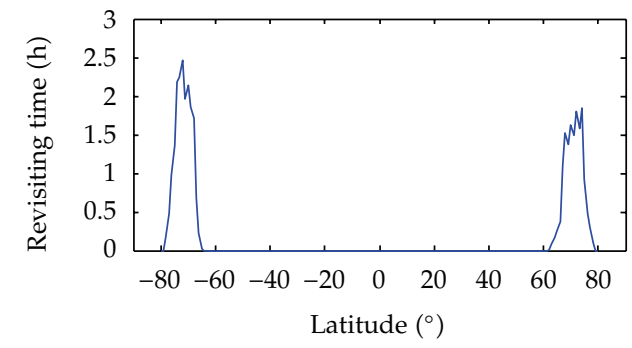

(g) Epoch time: 12:00, July 1, 2033

Figure 9: The full analysis on revisiting time on the Earth's surface during a whole Metonic cycle from 2015 to 2034 . The relationship between the latitudes and the revisiting times is implemented by the epoch times of (a) 12:00, July 1, 2015, (b) 12:00, July 1, 2018, (c) 12:00, July 1, 2021, (d) 12:00, July 1, 2024, (e) 12:00, July 1, 2027, (f) 12:00, July 1, 2030, and (g) 12:00, July 1, 2033.

4.2. Inserting Strategies and Costs into Nominal Trajectories. After being launching into the parking orbit around the Earth, some maneuvers are required to guidance the spacecrafts to nominal trajectories located near $L L_{2}, L L_{3}, L L_{4}$, and $L L_{5}$, respectively, which are refined in Section 2.2. Some inserting strategies are included in this paper to analyze the inserting costs for this constellation.

The invariant manifold theory developed in CR3BP has potential applications in creating the inserting trajectories into a halo orbit near $L_{1}$ point $[24,25]$. However, According to the technique developed by Koon et al. [26], the insertion into the quasi-periodic orbit near $L L_{2}$ point needs some combinations between unstable manifolds stemming from $E L_{1}$ (or $E L_{2}$ ) point and stable manifolds stemming from $L L_{2}$ point. In fact, the similar results can be achieved by the weak stability boundary theory (WSB) proposed by Belbruno and Miller [27] to create some low-energy cislunar trajectory with the help of the Sun's gravitation. Therefore, following on the initial guess obtained in CR3BP, the iteration procedure defined in Section 2.2 can be used to product the real inserting trajectory to the quasi-periodic orbit near $L L_{2}$ point in the full ephemeris model, shown in Figure 13. Only one maneuver is required on the parking orbit with its altitude of $200 \mathrm{~km}$ to insert the nominal orbit, without other 


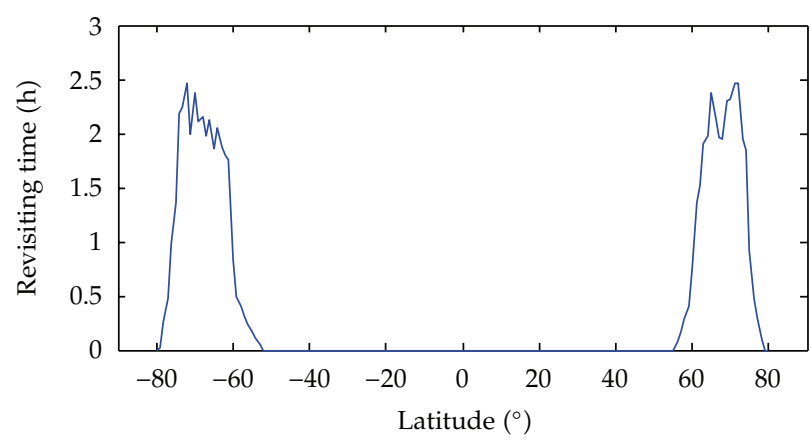

(a)

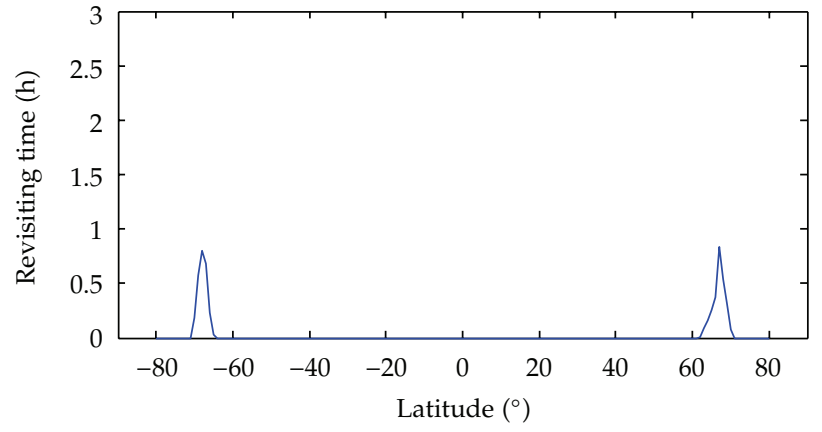

(b)

Figure 10: The relationship between the latitudes and the maximal and common revisiting times during a whole Metonic cycle from 2015 to 2034. The maximal revisiting times on the latitudes during a whole Metonic cycle are implemented in (a), and the minimal revisiting times in (b).

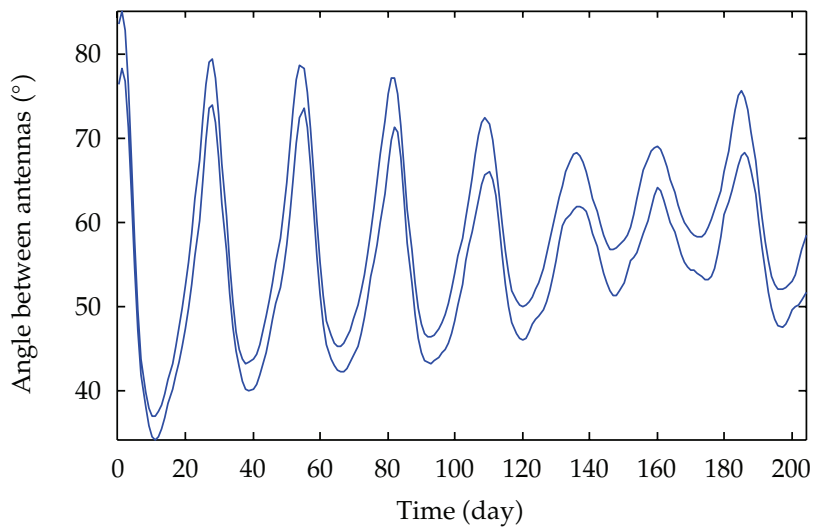

(a)

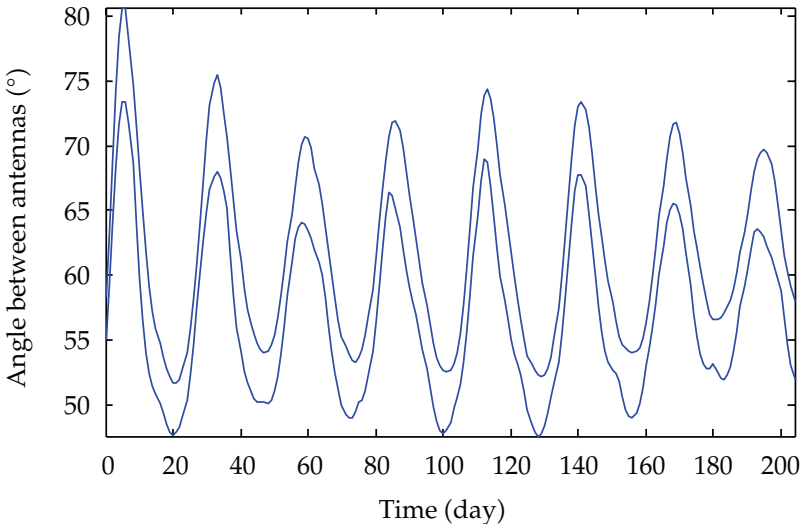

(b)

Figure 11: The angle between the two antennas equipped on the $L L_{4}$ and $L L_{5}$ spacecrafts. The maximal angle during a Metonic cycle is indicated by the upper boundary, while the minimal angle during a Metonic cycle is indicated by the lower boundary.

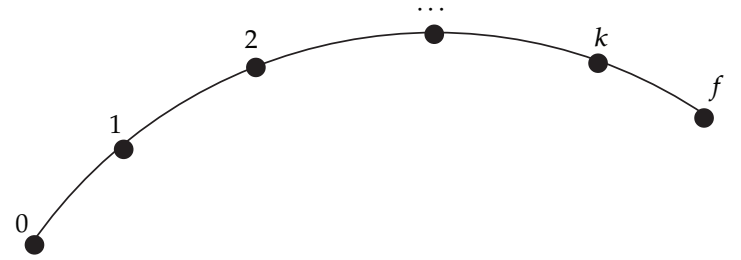

FIGURE 12: Stationkeeping corrections on the nominal trajectory.

maneuver at the end of the transfer trajectory. Thus, during this transfer, the total transfer duration is 91.3 days, and the total cost of maneuvers is $3.2018 \mathrm{~km} / \mathrm{s}$.

For the bounded trajectories near $L L_{3}, L L_{4}$, and $L L_{5}$, all of the stable manifolds stemming from these libration points are quite far from the Earth, which is different from $L L_{2}$ point. The Hohmann transfer is employed in this paper to guide the spacecrafts from the parking orbits to their stable manifolds, and then to insert the nominal trajectory following its stable manifolds. Based on the initial guess obtained in CR3BP, the iteration procedure defined in Section 2.2 can be used to product the real inserting trajectory to the bounded orbit near $L L_{3}, L L_{4}$, and $L L_{5}$ points in the full ephemeris model, shown in Figures 14, 15, and 16, respectively. Therefore, the whole transfer trajectory includes the Hohmann segment and coasting segment along the stable manifold, and two maneuvers are required on the parking orbit with its altitude of $200 \mathrm{~km}$ to insert the nominal orbit; the first one is imposed on the parking orbit, and the last one is imposed at the end of the Hohmann segment.

For $L L_{3}$ point, the total transfer duration is 45.1 days, and the total cost of maneuvers is $3.7518 \mathrm{~km} / \mathrm{s}$. For $L L_{4}$ point, the total transfer duration is 47.57 days, and the total cost of maneuvers is $3.7585 \mathrm{~km} / \mathrm{s}$. For $L L_{5}$ point, the total transfer duration is 63.4 days, and the total cost of maneuvers is $3.761 \mathrm{~km} / \mathrm{s}$. 


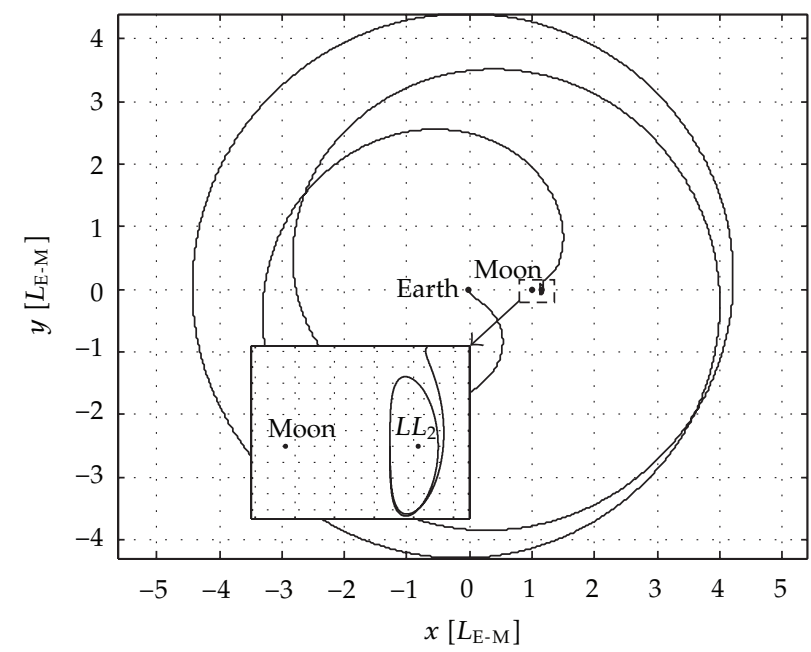

FIGURE 13: The inserting trajectory into the nominal orbit near $L L_{2}$ point from the parking orbit with its altitude of $200 \mathrm{~km}$ in the syzygy frame. The quasi-periodic halo orbit near $L L_{2}$ point created in full ephemeris model with the epoch time of 12:00, July 1, 2015. Only one maneuver $\Delta V=3.2018 \mathrm{~km} / \mathrm{s}$ on the parking orbit is required to insert the nominal orbit without other maneuvers at the end of the transfer trajectory. The transfer duration is 91.3 days.

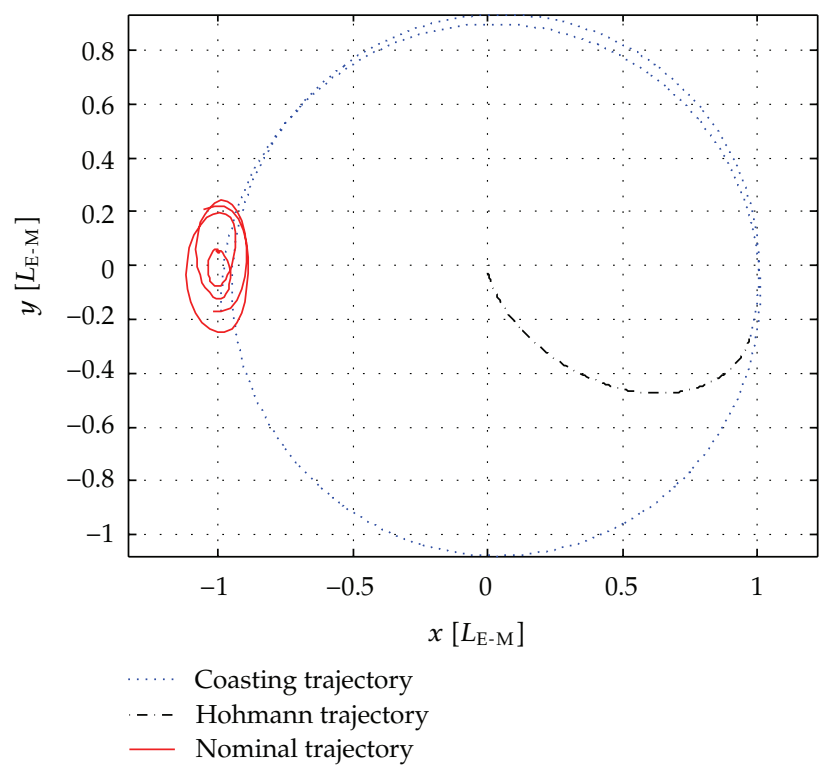

FIGURE 14: The inserting trajectory into the nominal orbit near $L L_{3}$ point from the parking orbit with its altitude of $200 \mathrm{~km}$ in the syzygy frame. The bounded orbit near $L L_{2}$ point created in full ephemeris model with the epoch time of 12:00, July 1, 2015. Two maneuvers of $3.7518 \mathrm{~km} / \mathrm{s}$ are required to insert the nominal orbit on the parking orbit and at the end of the Hohmann segment. The transfer duration is 45.1 days.

Therefore, the total inserting cost of this constellation is $14.4731 \mathrm{~km} / \mathrm{s}$, which is accepted for the aerospace engineering. Furthermore, the inserting costs by Hohmann segments in the cases of $L L_{3}, L L_{4}$, and $L L_{5}$ points can be optimized by some techniques developed in space manifold dynamics in the future.

\section{Communications}

There are several communicating links inside the constellation, which is shown in Table 2. The links make the constellation more functional than the single sum of the four satellites. Furthermore, the links make the constellation more robust, so the constellation will still work depending on the links at the event that one or two satellites happen to be broken.

The distance from Earth to Moon brings in a long time delay of $1.28 \mathrm{~s}$. Due to this, direct voice communications may be impossible. Instead, data communications are preferred for this constellation architecture, including some links between ground sites on the Earth and the far side on the Moon or some data exchanges between the points on the Earth, and so on. 
TABLE 2: Links inside the constellation ( $\sqrt{ }$ : link, $\times$ : no link).

\begin{tabular}{lcccccc}
\hline & $L L_{2}$ & $L L_{3}$ & $L L_{4}$ & $L L_{5}$ & Earth & Moon \\
\hline$L L_{2}$ & $\times$ & $\times$ & $\sqrt{ }$ & $\sqrt{ }$ & $\sqrt{ }$ \\
$L L_{3}$ & $\times$ & $\times$ & $\sqrt{ }$ & $\times$ & $\sqrt{ }$ \\
$L L_{4}$ & $\sqrt{ }$ & $\sqrt{ }$ & $\times$ & $\times$ & $\sqrt{ }$ & $\sqrt{ }$ \\
$L L_{5}$ & $\sqrt{ }$ & $\sqrt{ }$ & $\times$ & $\sqrt{ }$ & $\times$ \\
Earth & $\sqrt{ }$ & $\sqrt{ }$ & $\sqrt{ }$ & $\sqrt{ }$ \\
Moon & $\sqrt{ }$ & $\sqrt{ }$ & $\sqrt{ }$ & $\times$ \\
\hline
\end{tabular}

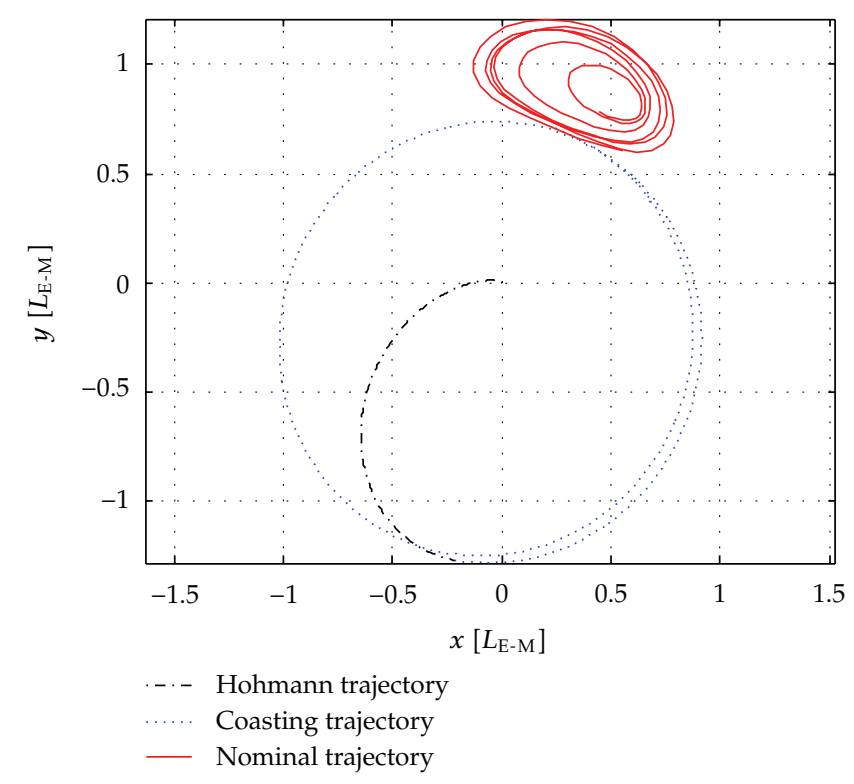

FIGURE 15: The inserting trajectory into the nominal orbit near $L L_{3}$ point from the parking orbit with its altitude of $200 \mathrm{~km}$ in the syzygy frame. The bounded orbit near $L L_{2}$ point created in full ephemeris model with the epoch time of 12:00, July 1, 2015. Two maneuvers of $3.7585 \mathrm{~km} / \mathrm{s}$ are required to insert the nominal orbit on the parking orbit and at the end of the Hohmann segment. The transfer duration is 47.57 days.

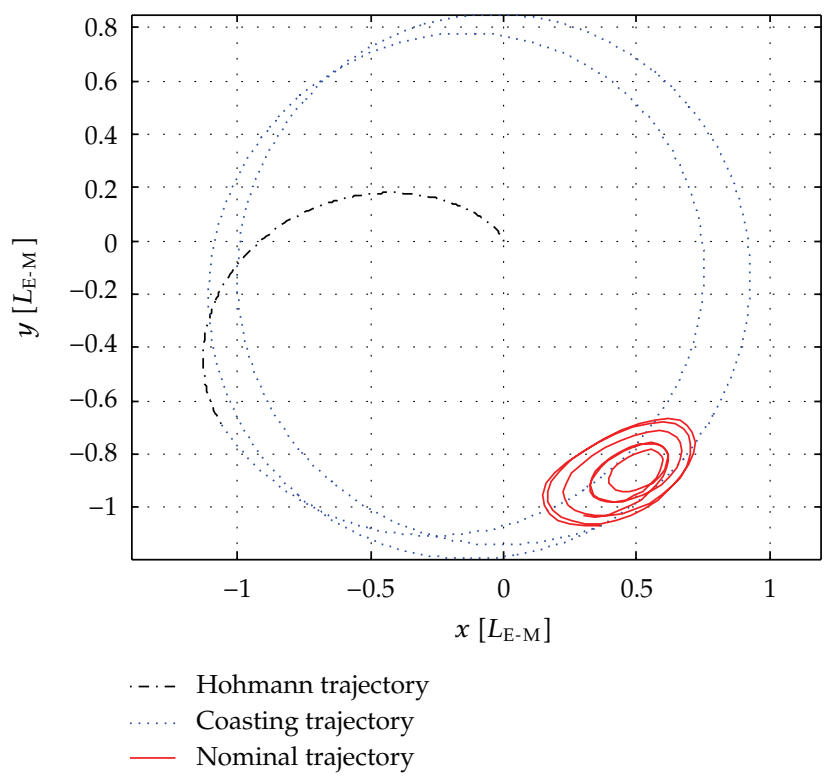

FIGURE 16: The inserting trajectory into the nominal orbit near $L L_{3}$ point from the parking orbit with its altitude of $200 \mathrm{~km}$ in the syzygy frame. The bounded orbit near $L L_{2}$ point created in full ephemeris model with the epoch time of 12:00, July 1, 2015. Two maneuvers of $3.761 \mathrm{~km} / \mathrm{s}$ are required to insert the nominal orbit on the parking orbit and at the end of the Hohmann segment. The transfer duration is 63.4 days. 
Furthermore, some ground stations are required to track the four spacecrafts in the constellation, and also to deal with orbital determination. Due to the data rates and Bands, the diameter of antenna is not less than $50 \mathrm{~m}$. According to the coverage region defined in Figure 8, the ground stations should be located at the latitudes from $80^{\circ} \mathrm{S}$ to $52^{\circ} \mathrm{S}$ and from $55^{\circ} \mathrm{N}$ to $79^{\circ} \mathrm{N}$ to track and manage this constellation.

\section{Conclusion}

To provide near-constant communications between any two points in the cislunar space, a new constellation configuration is proposed in this paper. Four satellites were allocated on some natural orbits near $L L_{2}, L L_{4}, L L_{5}$, and $L L_{3}$ points, the first three of which are expected to supply a global coverage on the Moon, while the last three afford a global coverage on the Earth. Considering the real dynamics and geometries, a full ephemeris model is employed to refine the quasi-periodic trajectories near $L L_{2}, L L_{3}, L L_{4}$, and $L L_{5}$ points by a multiple shooting corrector numerically.

The revisiting intervals between the successive revisits are demonstrated on the geographical maps of the Earth and the Moon, with a consideration of the Metonic cycle of the equatorial inclination with respect to lunar orbital plane. All the points on the lunar surface can be covered by this constellation, where most of them are accessed without any time interval, and few of them are revisited after some intervals less than 5 days. This constellation is weak in covering some longitude lines located near $150^{\circ} \mathrm{W}$ and $150^{\circ} \mathrm{E}$. The discontinuous communications are interrupted by some intervals less than 2 hours. Only some sites keep discontinuous communications during a whole Metonic cycle, which are located from $20^{\circ} \mathrm{N} 140^{\circ} \mathrm{W}$ to $70^{\circ} \mathrm{N} 140^{\circ} \mathrm{W}$, from $30^{\circ} \mathrm{S} 150^{\circ} \mathrm{W}$ to $2^{\circ} \mathrm{N} 150^{\circ} \mathrm{W}$, from $85^{\circ} \mathrm{S} 55^{\circ} \mathrm{E}$ to $85^{\circ} \mathrm{S} 100^{\circ} \mathrm{E}$, and from $80^{\circ} \mathrm{S} 170^{\circ} \mathrm{E}$ to $80^{\circ} \mathrm{S} 130^{\circ} \mathrm{W}$. The worst intervals occur at the high-latitude areas instead of the poles because the maximal revisiting time is 0.96 day at the north pole while 1.21 days at the south pole. This constellation has a good coverage performance at the lunar poles.

All the points on the Earth's surface can be covered by this constellation, and the discontinuous communications keep the invariant revisiting time on the same latitudes during a whole Metonic cycle. Few of regions located at the latitudes from $80^{\circ} \mathrm{S}$ to $52^{\circ} \mathrm{S}$ and from $55^{\circ} \mathrm{N}$ to $79^{\circ} \mathrm{N}$ are revisited after some intervals less than 2.5 hours, while the other regions are accessed without any time interval. The worst intervals occur at the regions located from $71^{\circ} \mathrm{S}$ to $65^{\circ} \mathrm{S}$ and from $62^{\circ} \mathrm{N}$ to $70^{\circ} \mathrm{N}$, which keep discontinuous communications during a whole Metonic cycle. This constellation has a good coverage performance at the Earth's poles with no time interval.

Moreover, cost analysis on stationkeeping and launching is implemented in detail in full ephemeris model. According to the stationkeeping and inserting strategies proposed in this paper, the total inserting cost of this constellation is $14.4731 \mathrm{~km} / \mathrm{s}$, and the total stationkeeping cost of this constellation is $261.8 \mathrm{~m} / \mathrm{s} /$ year.

Furthermore, some optimizations on this constellation can be implemented in the future, including optimizing the orbital elements in this constellation to promote the coverage on the Earth and Moon, selecting the correction schedule from viewpoint of the stochastic optimal control to decrease the stationkeeping cost, and optimizing the Hohmann segments for $L L_{3}, L L_{4}$, and $L L_{5}$ points to decrease the launching cost based on space manifold dynamics.

\section{Acknowledgments}

The research is supported by the National Natural Science Foundation of China (11172020); Talent Foundation supported by the Fundamental Research Funds for the Central Universities; Aerospace Science and Technology Innovation Foundation of China Aerospace Science Corporation; and the National High Technology Research and Development Program of China (863 Program: 2012AA120601).

\section{References}

[1] J. R. Wertz and J. W. Larson, Space Mission Analysis and Design, Microcosm Press and Kluwer Academic, Boston, Mass, USA, 3rd edition, 1999.

[2] J. G. Walker, "Some circular orbit patterns providing continuous whole earth coverage," Journal of British Interplanetary Society, vol. 24, no. 7, pp. 369-384, 1971.

[3] A. H. Ballard, "Rosette constellations of earth satellites," IEEE Transactions on Aerospace and Electronic Systems, vol. 16, no. 5, pp. 656-673, 1980.

[4] R. Farquhar, "Lunar communications with libration-point satellites," Journal of Spacecraft and Rockets, vol. 4, no. 10, pp. 13831384, 1967.

[5] R. Farquhar, "The utilization of Halo orbits in advanced lunar operations,” Rept. TND-365, NASA Goddard Space Flight Center, Greenbelt, Md, USA, 1971.

[6] N. Chow, E. Gralla, J. Chase, and N. J. Kasdin, "Low earth orbit constellation design using the earth-moon $L_{1}$ point," Advances in the Astronautical Sciences, vol. 119, pp. 2277-2294, 2005.

[7] D. Grebow, Generating periodic orbits in the circular restricted three-body problem with applications to lunar South Pole coverage [M.S. thesis], Purdue University, West Lafayette, Ind, USA, 2006.

[8] K. Bhasin and J. L. Hayden, "Developing architectures and technologies for an evolvable NASA space communication infrastructure," in Proceedings of the 22nd AIAA International Communications Satellite Systems Conference and Exhibit, pp. 1180-1191, Monterey, Calif, USA, May 2004.

[9] T. R. Kulkarni, A. Dharne, and D. Mortari, "Communication architecture and technologies for missions to moon, mars, and beyond," in Proceedings of the 1st Space Exploration Conference: Continuing the Voyage of Discovery, pp. 1210-1217, Orlando, Fla, USA, February 2005.

[10] S. Lee, J. H. Kim, and S. P. Lee, "Communications satellite system by two trojan and one equilibrium orbit satellites of the earth-moon system," in Proceedings of the 17th International Symposium on Space Flight Dynamics, Moscow, Russia, June 2003.

[11] S. Lee, J. H. Kim, and S. P. Lee, "Communications satellite system by using moon orbit satellite constellation," Journal of Astronautical Space Science, vol. 20, no. 4, pp. 313-318, 2003.

[12] D. J. Grebow, M. T. Ozimek, K. C. Howell, and D. C. Folta, "Multibody orbit architectures for lunar south pole coverage," Journal of Spacecraft and Rockets, vol. 45, no. 2, pp. 344-358, 2008. 
[13] M. Xu and $\mathrm{S} . \mathrm{Xu}$, " $J_{2}$ invariant relative orbits via differential correction algorithm," Acta Mechanica Sinica, vol. 23, no. 5, pp. 585-595, 2007.

[14] M. Xu, Y. Wang, and $\mathrm{S}$. Xu, "On the existence of $J_{2}$ invariant relative orbits from the dynamical system point of view," Celestial Mechanics \& Dynamical Astronomy, vol. 112, no. 4, pp. 427-444, 2012.

[15] M. Xu and $\mathrm{S} . \mathrm{Xu}$, "Nonlinear dynamical analysis for displaced orbits above a planet," Celestial Mechanics and Dynamical Astronomy, vol. 102, no. 4, pp. 327-353, 2008.

[16] M. Xu, Spacecraft orbital dynamics and control based on libration point theories [Ph.D dissertation], Beihang University, Beijing, China, 2008.

[17] D. L. Richardson, "Analytic construction of periodic orbits about the collinear points," Celestial Mechanics, vol. 22, no. 3, pp. 241-253, 1980.

[18] M. Popescu and V. Cardoş, "The domain of initial conditions for the class of three-dimensional Halo periodical orbits," Acta Astronautica, vol. 36, no. 4, pp. 193-196, 1995.

[19] D. Folta and F. Vaughn, "A survey of earth-moon libration orbits: stationkeeping strategies and intra-orbit transfers," in AIAA/AAS Astrodynamics Specialist Conference, pp. 87-106, Providence, RI, USA, August 2004, AIAA 2004-4741.

[20] M. Xu and S. Xu, "Study on stationkeeping for Halo orbits at $E L_{1}$ : dynamics modeling and controller designing," Transactions of the Japan Society for Aeronautical and Space Sciences, vol. 55, no. 5, pp. 274-285, 2012.

[21] G. Gómez, K. C. Howell, J. Masdemont, and C. Simo, "Station-keeping strategies for translunar libration point orbits," Advances in the Astronautical Sciences, vol. 99, no. 2, pp. 949969, 1998.

[22] M. Xu and S. Xu, "Trajectory and correction maneuver during the transfer from Earth to Halo orbit," Chinese Journal of Aeronautics, vol. 21, no. 3, pp. 200-206, 2008.

[23] M. Xu, T. Tan, and S. Xu, "Stochastic optimal maneuver strategies for transfer trajectories," Journal of Aerospace Engineering. In press.

[24] G. Gómez, A. Jorba, C. Simó, and J. Masdemont, Dynamics and Mission Design Near Libration Points.Vol. IV Advanced Methods for Triangular Libration Points, vol. 5 of World Scientific Monograph Series in Mathematics, World Scientific, River Edge, NJ, USA, 2001.

[25] M. Xu, T. Tan, and $\mathrm{S}$. Xu, "Research on the transfers to Halo orbits from the view of invariant manifolds," Science China Physics, Mechanics and Astronomy, vol. 55, no. 4, pp. 671-683, 2012.

[26] W. S. Koon, M. W. Lo, J. E. Marsden, and S. D. Ross, "Low energy transfer to the Moon," Celestial Mechanics and Dynamical Astronomy, vol. 81, no. 1-2, pp. 63-73, 2001.

[27] E. A. Belbruno and J. K. Miller, "Sun-perturbed earth-to-moon transfers with ballistic capture," Journal of Guidance, Control, and Dynamics, vol. 16, no. 4, pp. 770-775, 1993. 


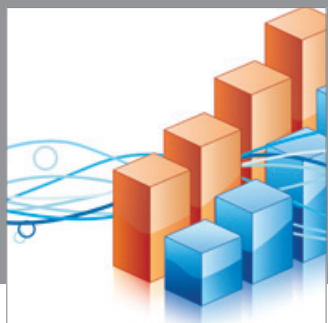

Advances in

Operations Research

mansans

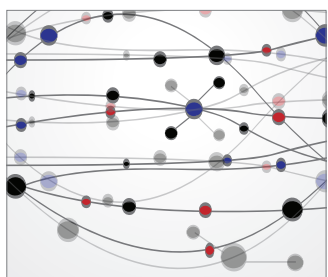

The Scientific World Journal
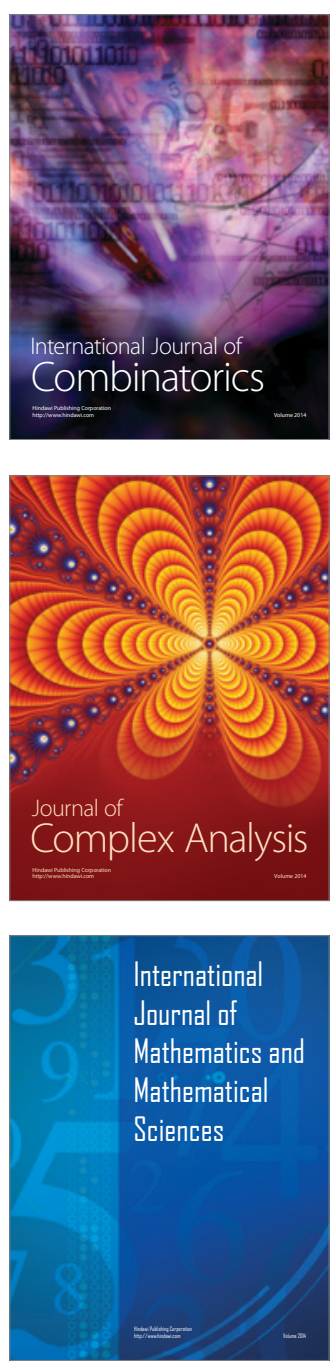
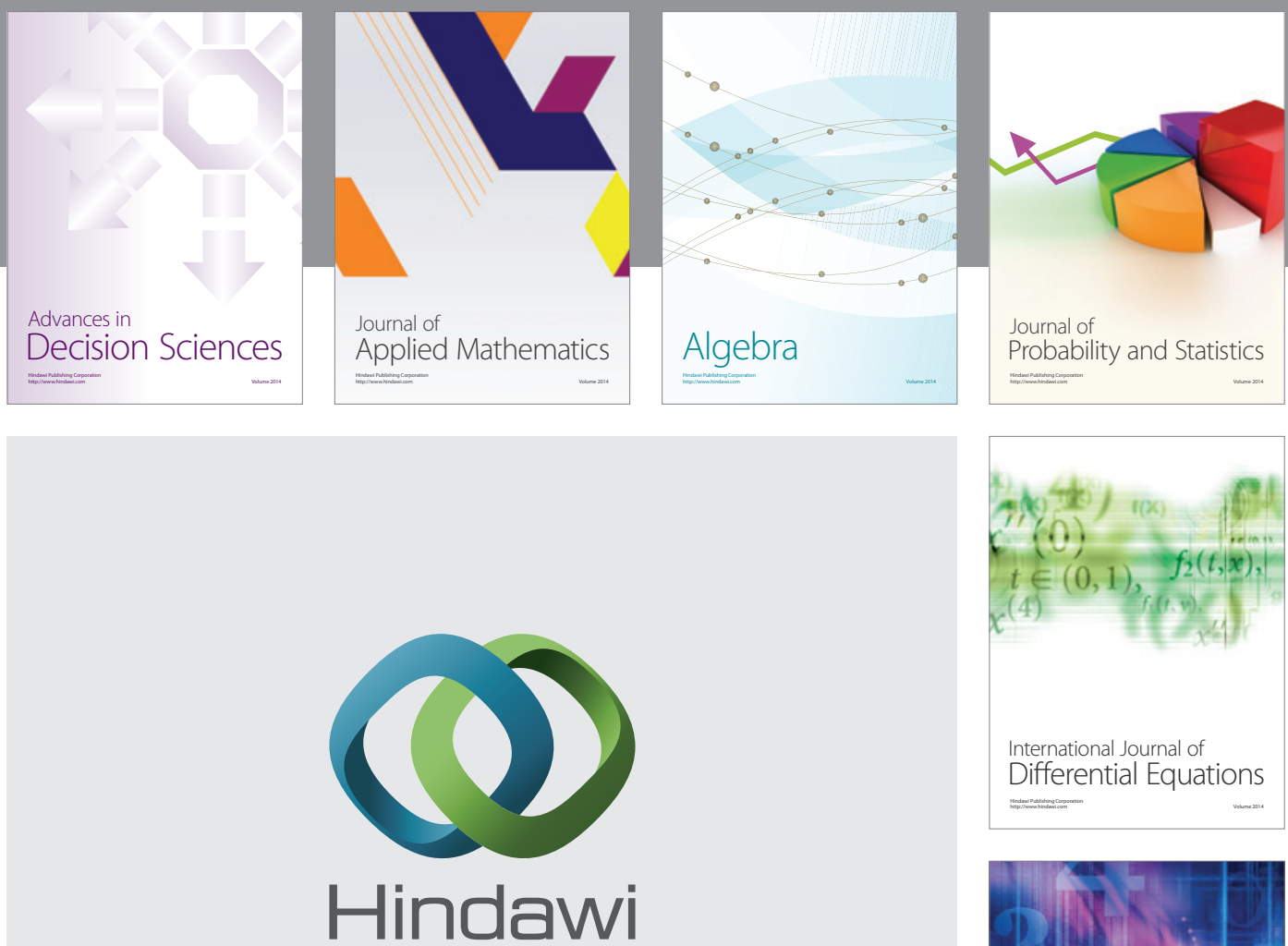

Submit your manuscripts at http://www.hindawi.com
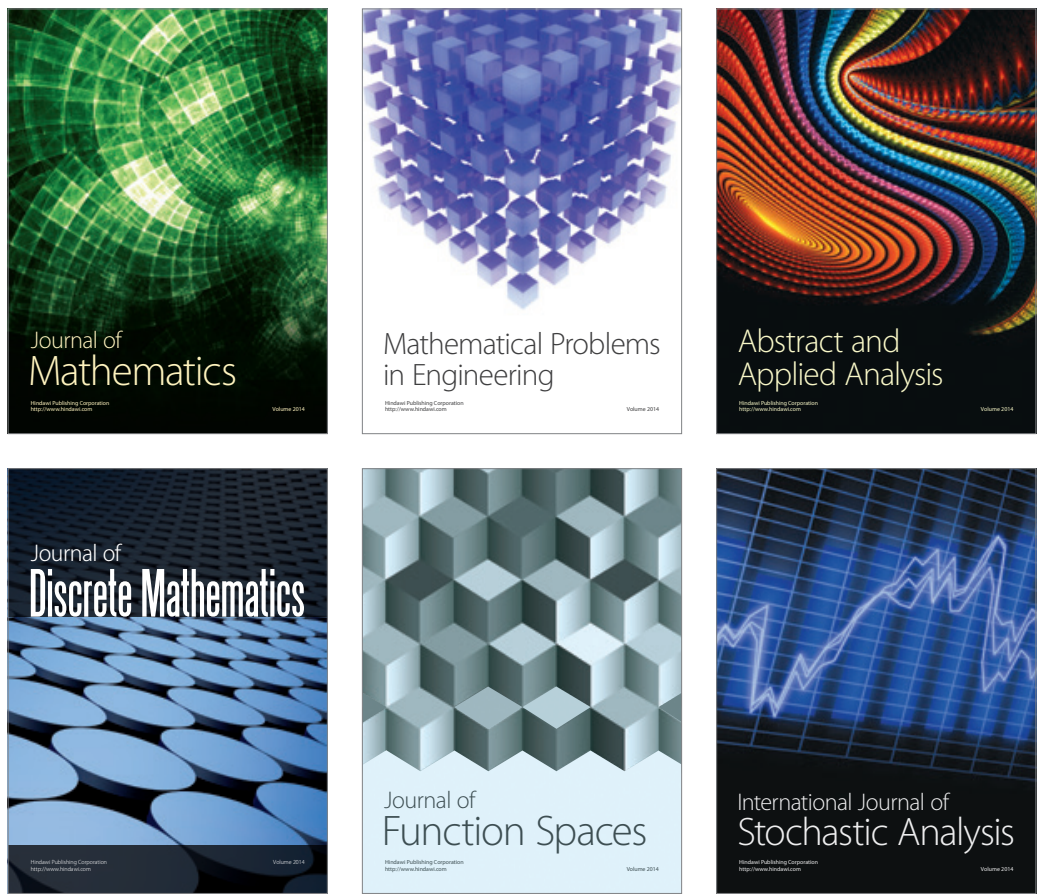

Journal of

Function Spaces

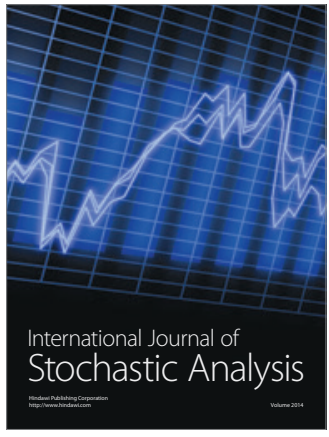

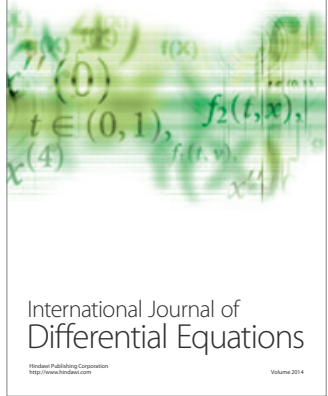
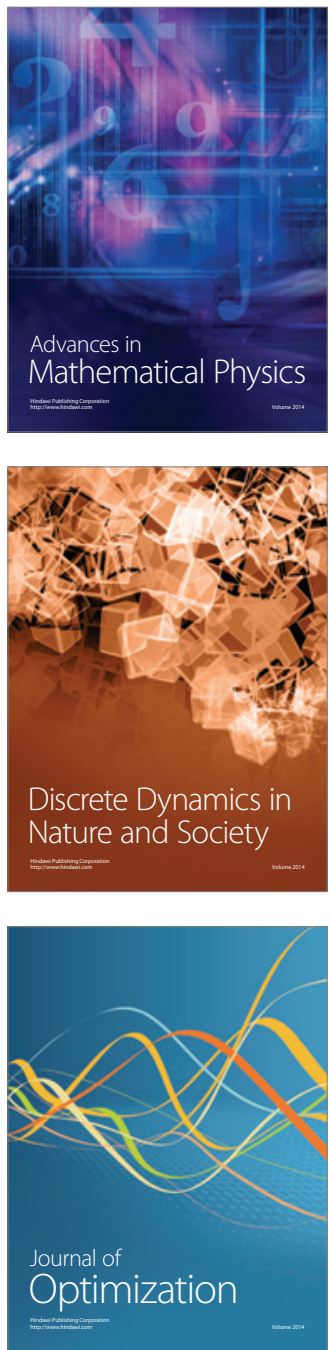